at the end. Outline solutions to the problems are given at the end of the book. Printing and diagrams are excellent.

Chapter one, a short one, deals in an introductory way with Fermat's principle. The second chapter introduces Hamilton's point characteristic $V$ and the general notion of a characteristic function, then the angle characteristic $T$, the "mixed" characteristics $W_{1}$ and $W_{2}$, and finally the notion of an aborration function. Chapter three discusses the relation between symmetries of optical systems and invariance properties of characteristic functions. Ordinary centred systems, of course, provide the most important practical examples, but the discussion is more general. Chapters four and five develop the theory of the symmetric system (the ordinary "centred system") and chapter six that of systems with additional symmetries. Chapter seven discusses semi-symmetric systems, which have an axis of symmetry but no plane of symmetry containing the axis. Here the medium is necessarily anisotropic. (Electron microscopes using magnetic focusing provide an example of this type of system.) Systems with other types of symmetry are discussed more briefly in the following two chapters. In chapter ten, a fairly short discussion of chromatic defects of the image is given. Chapter eleven deals with the effects of anisotropy, such as can occur in crystalline media.

The last chapter, on the computation of aberration coefficients, begins with an interesting discussion on whether the Hamiltonian or the Lagrangian method of computation should be considered the best practical basis for optical (and dynamical) computations. So far as optical computations are concerned, the author is probably better qualified than most experts to pass judgment on this question. He says: "The great simplicity of the Lagrangian method leads me to believe that it, rather than the Hamiltonian method, is best adapted to the problem of practical calculations; though this is admit. tedly a mero expression of opinion". Nevertheless, because of the interest of this question and also to round off the treatment, he goes on to give one possible procedure of calculating characteristic functions, that is to say the coefficients of their power series.

The book is an outstanding contribution to the "classical" literature of the subject and deserves a place in every optics library.

E. H. Linfoot

\section{ABELIAN GROUPS}

Infinite Abelian Groups

By Laszlo Fuchs. Vol. 1. (Pure and Applied Mathematics, Vol. 36.) Pp. xi +290 . (Academic Press: New York and London, January 1970.) 140s.

THe rate at which group theory is growing is indicated by the fact that the author, intending to prepare a revised edition of his 1958 book on Abelian groups, found that in order to take reasonable account of work done during the past decade a completely new book must be written. His new volume should therefore be particularly useful to the young postgraduate student, for in many places it brings him up to the frontiers of present-day knowledge, shows him modern methods and, in addition, offers a number of explicit research problems to challenge him. The reader should have some knowledge of abstract algebra, set theory, and topolegy; from this point onwards the book is self-contained, but a previous acquaintance with the fundamental properties of Abelian groups and with the type of austerely abstract reasoning involved, while not absolutely essential, is highly desirable. The author has striven for clarity of expression, and has not thought the provision of numerous exercises for the reader beneath the dignity of a volume intended for postgraduate study; these are genuine exercises, not sections of theory for which no room could be found in the main text.

The influence of homological algebra is evident; the 1956 book on this topic by Cartan and Eilenberg unified cohomology theory for groups and classes of algebras and helped to clarify and simplify certain aspects of group theory. The concepts of categories and functors supply generality, and the diagrammatic representation of homomorphisms gives an intuitive grasp of complex relationships.

The first volume deals chiefly with important classes of Abelian groups, direct sums, dirisible groups, and the like, and extension problems and torsion products. The second volume is to deal with structure theory and applications. T. A. A. Broadbent?

\section{HYDRATION PROCESSES}

Hydration and Intermolecular Interaction

Infrared Investigation with Polvelectrolyte Membranes. By Georg Zundel. Pp. xi+310. (Academic Press: Now York and London, January 1970.) $168 s$.

THIS volume summarizes the results of investigations by infrared spectroscopy carried out at the University of Munich on the molecular processes involved in hydration, particularly of polyelectrolytes in the form of cationexchange resins.

The assignment of the absorption bands of polystyrene, polystyrenylsulphonyl chloride and of polystyrene-sulphonic, -selenoic, -phosphinic and thiophosphonic acids and their salts is fundamental to the interpretation of the measurements, and is therefore analysed critically. A careful examination is also made of the effect of hydrogen-bonding on the spectra of water and deuterated water, as well as of such phenomena as the cffects of the location of the cation with respect to the oxygen atoms of the anion and of the degree of hydration on the stretching vibrational frequencies of polyelectrolyte anions. The effects of hydration and of association of acid groups on the spectra of the acids are collected and discussed. It is concluded that the two bands always occurring, which must be ascribed to $\mathrm{OH}$ or OD groups in the hydrogenbridges connecting the acid groups, cannot be caused by a double minimum in the potential energy curve, but arise from a stretching vibration and an overtone of the bending vibration of the OH and OD groups. The natures of the various forms of hydrated proton which occur in these systems and the tunnelling effects in proton migration are discussod. Finally, the book gives details of the apparatus and techniques used in preparing cation-exchange mem. branes by production of a polystyrene nembrane of definite thickness and degree of cross-linking and subsequent introduction of the desired acidic groups. The techniques used in the investigation of such membranes by infrared spectroscopy are also discussed.

The book provides an extremely important summary of the subject for those interested in the structures of these systems, but its price will seem high to British readers. J. W. SurTH

\section{CHEMICAL MARINE BIOLOGY}

\section{The Chemical Biology of Fishes}

With a Key to the Chemical Literature. By R. Malcolm Love. Pp. $x v+547$. (Academic Press: London and New York, January 1970.) 140s; $\$ 21$.

Changes in the external enviromment such as those of salinity, oxygen tension, temperature and pressure pose special problems to the maintenance of a state of homeostasis (constancy of internal environment) by fishes over the whole life eycle. This book provides a much needed survey and discussion on the chemical composition of tissues and body fluids of wild fish. as well as of fish that 